\title{
Exploration Decisions Precede and Improve Explicit Uncertainty Judgments in Preschoolers
}

\author{
Elizabeth Lapidow (elapidow@ucsd.edu) \\ Department of Psychology, University of California, San Diego \\ Isabella Killeen (kille093@umn.edu) \\ University of Minnesota Law \\ Caren M. Walker (carenwalker@ucsd.edu) \\ Department of Psychology, University of California, San Diego
}

\begin{abstract}
We investigate the relationship between exploratory learning and confidence scale judgments in understanding and improving children's early recognition of uncertainty. Fourand five-year-olds were presented with stimuli that varied in their amount of occlusion. We assessed children's ability to distinguish between these levels of uncertainty using two types of measures. Experiment 1 used a traditional 3-point confidence scale to examine explicit uncertainty judgments. Experiment 2 examined exploration preference as an implicit measure of uncertainty using the same stimuli. We compared children's performance on these two tasks before and after their experience of disconfirming evidence, to assess the impact of surprising events on the recognition of uncertainty. Results indicate that children intuitively recognize gaps in their knowledge and express this in their exploratory behavior before they are able to spontaneously produce accurate confidence judgments. We also find that this implicit recognition of uncertainty may be leveraged to support and improve explicit judgments, even without extensive training.
\end{abstract}

Keywords: cognitive development; confidence judgments; exploration; uncertainty monitoring; decision-making; ambiguity

\section{Introduction}

Children under eight years of age have traditionally been described as 'eternal optimists' who tend to express overconfidence in their judgments (Newman \& Wick, 1987; Beck, et al., 2011). That is, children often indicate high confidence even when they are likely to be incorrect, based on the level of uncertainty present (Roebers, 2002), and also tend to treat partial knowledge as complete (Rohwer, et al., 2012).

This apparent insensitivity to uncertainty contrasts with claims that spontaneous exploration in early childhood is guided by a preference for what is unknown, uncertain, or unusual, which supports self-directed learning. Specifically, children attend to and preferentially explore instances where they have incomplete or inconsistent knowledge (e.g., Legare, 2012; Schulz \& Bonawitz, 2007; Bonawitz, et al., 2012 ), select actions with the potential to improve their epistemic status (e.g., Stahl \& Feigensen, 2017; Schulz \& Bonawitz, 2007), and increase their tendency to test their hypotheses when confronted with surprising or inconsistent data (e.g., Cook, et al., 2011; Lapidow \& Walker, 2020). This evidence not only demonstrates that children intuitively recognize gaps in their knowledge, but also shows that this recognition can motivate decision-making behavior from a young age.

\section{Implicit and Explicit Measures of Uncertainty}

One proposal explaining the contrast between these literatures emphasizes a distinction between children's implicit and explicit awareness of uncertainty (Ghetti, et al., 2013). Research from Ghetti and colleagues has shown that, by preschool, children begin to introspect on their subjective feelings of uncertainty with some accuracy (Hembacher \& Ghetti, 2014; Destan, et al., 2014; Coughlin, et al., 2015; Lyons \& Ghetti, 2011; 2013). This early awareness is observed in children's developing ability to engage in "uncertainty monitoring"- the introspective process by which a learner considers whether a decision made under unreliable conditions is likely to be correct (Ghetti et al., 2013). This is typically measured by asking children to explicitly report how 'sure' they are about an outcome or (more often) a judgment, using a scale with two or three fixed-points, corresponding to greater or lesser confidence (Lyons \& Ghetti, 2011; Hembacher \& Ghetti, 2014). Uncertainty monitoring is indicated when the average confidence rating given for correct judgments is higher than for incorrect judgments.

However, these studies have also shown that children's sensitivity to uncertainty can be measured indirectly via uncertainty control-using the output of metacognitive monitoring to regulate and optimize decision-making behavior when feeling uncertain (Koriat \& Goldsmith, 1996; Ghetti, et al., 2013). This ability has been found in a number of different behaviors, including the amount of selfallocated study time (Destan et al., 2014), the tendency to seek help (Coughlin et al., 2015), the decision not to have one's judgment assessed (Hembacher \& Ghetti, 2014), and opting-out of making the judgment at all (Lyons \& Ghetti, 2013). For example, Hembacher and Ghetti (2014) asked children to explicitly rate their confidence in their own recognition judgments of previously studied items using a 3- 
point scale. They then compared these ratings to children's decisions about whether or not to have the experimenter check the accuracy of those judgments. They found that 4and 5-year-olds' average confidence was somewhat higher for accurately identified items, but that the relationship between their accuracy and their willingness to have their judgments evaluated was far more robust. From this, the authors argued that the connection between uncertainty and decision-making might precede the ability to explicitly monitor and report these feelings in childhood (Hembacher \& Ghetti, 2014).

\section{The Current Study}

We aim to extend the investigation of implicit and explicit awareness of uncertainty in early childhood by examining the relationship between exploratory behavior and confidence judgments. To do this, we developed a novel set of stimuli that manipulated the level of uncertainty present. On each trial, children observed three "windows" that differed in their degree of occlusion of a target shape (see Figure 1). The 'Clear' window was an empty frame, leaving the shape entirely visible and unambiguous. The frame of the 'Partial' window included a cross bar, leaving a portion of the shape concealed. The 'Full' window was entirely obscured. Thus, on each trial, we presented children with three distinct levels of ambiguity about the identity of the shape inside (i.e., whether the window contained the target shape or not), and asked whether they could accurately distinguish among them in explicit (Experiment 1) and implicit (Experiment 2) measures of uncertainty.

In Experiment 1, we first assess children's baseline ability to spontaneously use a 3-point confidence scale to indicate their confidence that the target shape was located behind each window. Prior studies examining uncertainty monitoring in preschoolers have all relied on the presentation of multiple training trials to calibrate their expression of confidence (e.g., Lyons \& Ghetti, 2013; Hembacher \& Ghetti, 2014). As the trials progress, children become increasingly sensitive to the differences between confidence levels, calibrating their scale use through a process of trial and error. It therefore remains unknown whether untrained preschool-aged children can use a confidence scale correctly before acquiring task-specific experience.

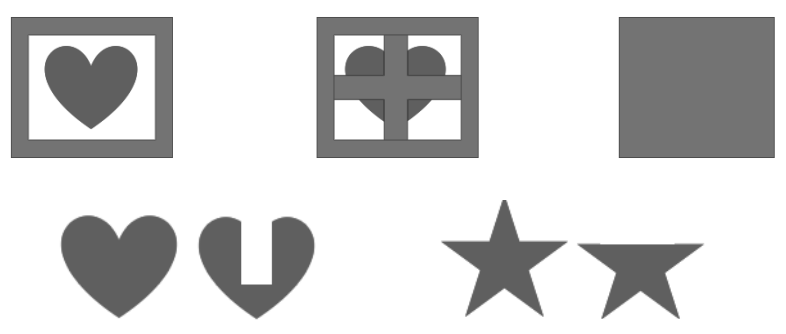

Figure 1: Task stimuli. Top row: 'Windows' (Clear, Partial, and Full) with heart as target shape. Bottom row: Examples of 'target' and 'non-target' shapes for heart and star.
To address this, we used a scale modeled on Hembacher and Ghetti (2014), which asks children to report how 'sure' they are on a three-point scale (i.e., "not sure," "a little bit sure," or "very sure"). Sensitivity to uncertainty is indicated when children use the different scale values to appropriately distinguish among the levels of ambiguity present. For example, when confronted with the Clear window, which provides direct, unambiguous evidence about the identity of the shape inside, children should be more likely to rate their confidence as "very sure." On the other hand, when confronted with a window providing no or incomplete evidence, children should be more likely to rate their confidence about the identity of the shape inside as "not sure" or only "a little bit sure."

In addition to establishing their untrained baseline performance, we also presented children with disconfirming evidence on either the first or second trial of the task to determine whether implicit sensitivity to surprising events may be leveraged to improve their explicit confidence judgments. The crossbar of the Partial window frame was placed so that the identity of the shape inside appears obvious (see Figure 1). Unknown to the participant, however, the shapes placed behind this window were sometimes altered: portions of the target shapes were cut out in such a way to be completely concealed behind the crossbar. When the shape was subsequently revealed, therefore, it violated their (reasonable) expectation that it was the target, disconfirming their initial hypothesis about the true contents of the window.

Given children's sensitivity to surprising outcomes during exploratory learning, we predict that observing these events may cue them to the presence of uncertainty in the environment, and improve their use of the confidence scale on subsequent trials, even in the absence of direct feedback. Indeed, Ghetti, Hembacher, and Coughlin (2013) proposed that the ability to introspect on one's own uncertainty may emerge from children's repeated exposure to uncertain situations or outcomes, which in turn supports the development of conceptual understanding. However, to our knowledge, our study is the first to test this claim directly.

Next, Experiment 2 uses a modified version of this task to examine children's exploration of the same set of stimuli as an implicit measure of their sensitivity to uncertainty. We can thus compare children's information-seeking decisions to their explicit confidence judgments from Experiment 1. Specifically, rather than asking children to report their uncertainty about each window, Experiment 2 offers a forced choice to reveal the contents of one of the three windows in order to learn more about the shape inside.

The decision to seek additional information has previously been used as an indication of uncertainty control in infants (Kidd, et al., 2012) and non-human primates (e.g., Beran, et al., 2013; Paukner, et al., 2006; Marsh \& MacDonald, 2012), but has never been compared to explicit uncertainty judgments. For example, Call and Carpenter (2001), presented orangutans, chimpanzees, and 2-year-old children with a task in which food was placed inside one of 
two hollow tubes, which the participant would be awarded if they selected correctly. On trials where food placement was occluded, apes and children were more likely to look into the tubes before making their choice, relative to trials where food placement was known.

Given this prior work, we predict that children will show earlier sensitivity to uncertainty in their implicit information seeking decisions relative to their explicit confidence judgments for the same set of stimuli. Further, we examine whether belief-violating evidence might scaffold children's ability to calibrate their expressions of confidence to better reflect this uncertainty. If so, this would raise the possibility that children's early experience with uncertainty may play a role in the later development of metacognition.

\section{Experiment 1}

\section{Method}

Participants A total of 43 children participated in Experiment 1, including 21 4-year-olds, $(M=54.52$ months, $S D=5.57$, range: $49-59$ months) and 22 5-year-olds $(M=$ 64.82 months, $S D=3.75$, range: 60-71 months). An additional 22 children were excluded, due to failure to pass the comprehension check (6), experimenter error (9), caretaker interference (2), or failure to complete the entire task (5). Children were recruited from local preschools and science museums. While specific demographic information was not collected from individual participants, demographics of the recruitment locations suggest the participants were predominately white $(44.5 \%)$ and middleclass (median household income of $\$ 73,900)$.

Materials Two confidence scales were used. Half of participants were randomly assigned to receive a newly developed 3-point confidence scale using photographs of a child displaying facial expressions and body language for each level of confidence, and half received a standard, 3point "smiley face" scale. ${ }^{1}$

The "windows" were constructed using two sheets of paper (one white, one blue), inserted into a clear plastic sheet protector. The bottom, white sheet was used to create a solid background for each of the windows. The top blue sheet was either left intact (for the Full window) or cut to resemble an open or bisected frame (for the Clear and Partial windows) (see Figure 1).

Paper shapes could be placed inside the windows by sliding them between the top and bottom sheets. Two types of paper shapes (circles, hearts, squares, and rectangles) were created. 'Targets,' included standard instances of each shape, and 'non-targets,' included each shape with a part cut out (see Figure 1). The cut outs were placed such that the removed portion would be hidden behind the crossbar of the Partial windows. This meant that target and non-targets would look identical at this level of occlusion. Reference cards with images of the target shapes, of the same size and

\footnotetext{
${ }^{1}$ No differences in performance were found between the two scales, so data were combined for all analyses.
}

color as those to be placed inside the windows, were also used.

Procedure Children were tested one-on-one with the experimenter. At the start of the task, the experimenter presented three empty windows (Full, Partial, and Clear), and arranged them in a row on the table. The order of the windows on the table was randomized and counterbalanced across trials and participants. The experimenter introduced the windows and explained that shapes can be placed inside them. To demonstrate, the experimenter showed the child three identical paper shapes, and placed them, one by one, inside each of the windows. This allowed children to observe what the same shape looked like behind each level of occlusion.

Children were also introduced to the confidence scale. Following previous work, they were instructed to point to the image that represented how sure they were (i.e., "not sure," "a little bit sure," or "very sure"). To ensure children understood the task, they were asked, "Which one do you point to when you're [very, a little bit, not] sure?" for all three levels of confidence. If children were unable to complete this task, their data was excluded from further analysis $(n=6)$.

Test Trials. Following this training, three test trials were conducted. At the start of Trial 1, the experimenter produced three new windows (Full, Partial, and Clear), with a set of paper shapes already inside them. The experimenter laid the windows on the table, held up the reference card with an image of the target-shape, and said, "some of these windows have a [shape name] like this behind them, and some of these windows have a different shape behind them." The experimenter asked participants to use the 3-point confidence scale to indicate their certainty that the target shape was behind each window, in turn. For each window, the experimenter would repeat the question and name the points by saying: "Are you very sure, a little bit sure, or not sure at all?" while pointing to the corresponding image on the scale. Children were instructed to respond by pointing to one of the images on the scale.

After children produced baseline confidence judgments for all three windows on Trial 1, the experimenter revealed each of the concealed shapes in turn. Two target shapes and one non-target shape were revealed on each trial. The Clear window always contained a target shape, and the non-target shape was revealed to be inside either the Partial or the Full window. "Disconfirming evidence" was defined as revealing a non-target shape from behind the Partial window, which violated children's (reasonable) expectation that there was a target shape in this location. In order to avoid biasing children to believe that Partial windows always contained non-target shapes, this disconfirming evidence was presented on only one of the first two trials (on the other trial, the non-target was located behind the Full window), with the order of presentation alternating across participants. 
After revealing each shape, the experimenter then removed the windows, shapes, and reference card from the table and moved on to the next trial. Trials 2 and 3 were then conducted using the identical procedure, save that each trial used a different target-shape.

\section{Results}

To examine the extent to which children's confidence judgments were sensitive to the different levels of uncertainty created by shape occlusion, we coded scale responses numerically and calculated mean confidence scores for each of the windows (following Hembacher \& Ghetti, 2014). 'Very sure,' or high-confidence responses, were coded as 3, 'little bit sure,' or medium-confidence responses, as 2, and 'not sure at all,' low-confidence responses, were coded as 1 .

Baseline Confidence Judgments On Trial 1, collapsing across age groups, children spontaneously reported significantly lower average confidence for the Full window $(M=2.4, S D=0.7)$ than the Clear window $(M=2.7, S D=$ $0.6), t(84)=-2.04, p=0.044$. Average confidence for the Partial window fell in between $(M=2.6, S D=0.67)$, and did not differ from either the Full, $t(84)=1.36, p=0.176$, or Clear windows, $t(84)=-0.64, p=0.523$.

However, when examining each age group individually, two distinct patterns of responding emerged (see Figure 2). Four-year-olds showed no differences in average confidence across any of the windows (Full: $M=2.4, S D=0.7$; Partial: $M=2.7, S D=0.7$; Clear: $M=2.5, S D=0.8$; all $p$-values greater than 0.10). Five-year-olds average confidence ratings, by contrast, were significantly higher for the Clear window $(M=2.9, S D=0.3)$ than for either the Full $(M=$ $2.3, S D=0.8), t(42)=-2.99, p=0.005$ or Partial windows $(M=2.4, S D=0.7), t(42)=-2.35, p=0.024$. However, average confidence rating for the Full and Partial windows did not differ, $t(42)=0.6, p=0.555$.

Confidence Judgments following Disconfirming Evidence We were also interested in the effect of disconfirming evidence on children's confidence scale use. Comparing responses on Trials 1 and 3 across ages revealed that, as predicted, average confidence ratings for the Full, $t(84)=2.468, p=0.016$, and Partial, $t(84)=2.401, p=$ 0.018 windows were significantly lower after observing disconfirming evidence. Average confidence for the Clear window did not differ, $t(84)=-0.894, p=0.374 .^{2}$

Analysis within each age group indicates that this result is driven by improvements in four-year-olds. Five-year-olds' pattern of average confidence did not change between the first and third trials (all $p$-values greater than 0.20). Consistent with their baseline performance, they gave significantly higher confidence ratings for the Clear window $(M=2.9, S D=0.5)$ than for either the Full $(M=2, S D=$ $0.9 ; t(42)=-3.91, p=0)$ or the Partial $(M=2.1, S D=0.9$;

\footnotetext{
${ }^{2}$ Whether children observed disconfirming evidence on the first or second Trial had no effect on how their confidence judgments for any of the windows on Trial 3 (all $p \mathrm{~s}>0.5$ ).
}

$t(42)=-3.4, p=0.002)$ windows, and did not distinguish between the Full and Partial windows, $t(42)=0.5, p=$ 0.621 . On the other hand, 4-year-olds' confidence ratings were significantly lower on Trial 3 than on Trial 1 for both the Full $t(40)=2.345, p=0.024$ and Partial $t(40)=2.177, p$ $=0.0354$ windows. That is, following their experience of disconfirming evidence, younger children's performance showed a similar pattern to that seen in older children at baseline: Significantly higher confidence ratings for the Clear window $(M=2.7, S D=0.6)$ than for either the Full $(M=1.9, S D=0.8), t(40)=-3.7, p=0.001$, or the Partial windows $(M=2.2, S D=0.8), t(40)=-2.18, p=0.035$. Again, the difference between ratings for Full and Partial windows was not significant, $t(40)=1.41, p=0.168$.

\section{Discussion}

Experiment 1 examined 4- and 5-year-olds explicit uncertainty monitoring by looking at average confidence scale ratings before and after observing surprising outcomes. On Trial 1, younger children's confidence ratings did not indicate sensitivity to the different levels of uncertainty: they gave roughly the same confidence rating in response to all three windows. Five-year-olds, by contrast,
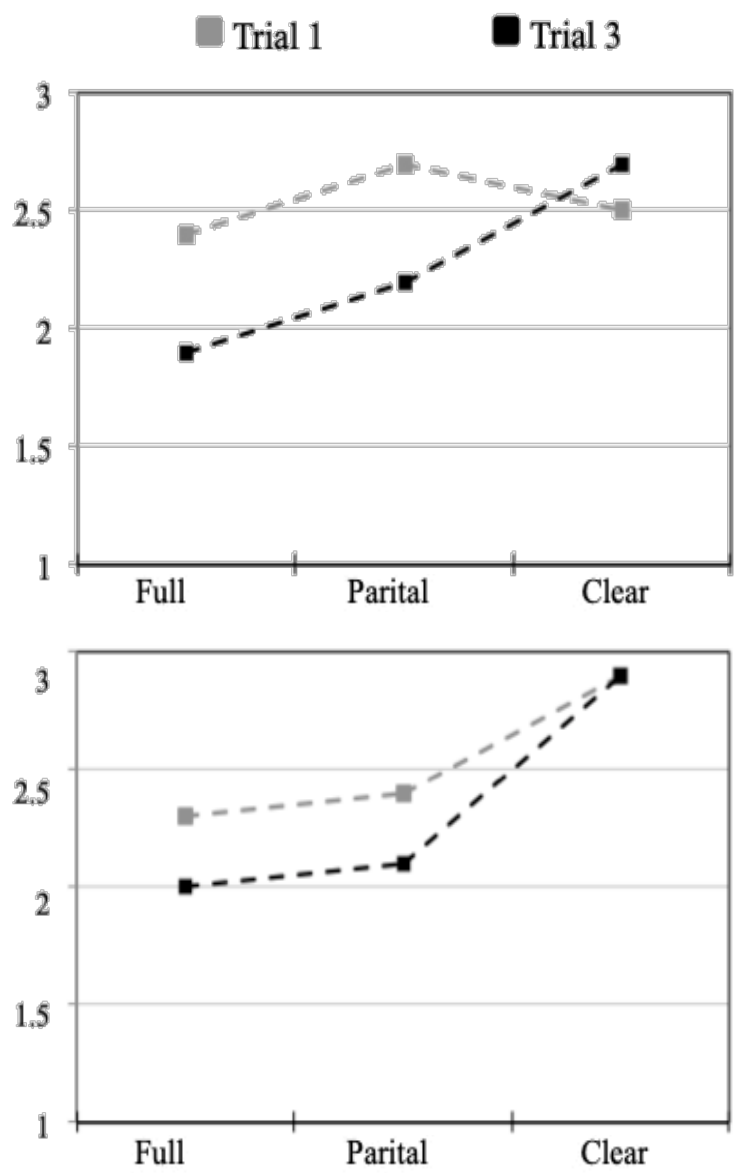

Figure 2. Average confidence ratings in Experiment 1 for four-year-olds (top) and five-year-olds (bottom). 
spontaneously distinguished between the maximally uncertain (Full) and maximally certain (Clear) windows, and also between the Partial and Full windows. However, not even the older children's responses distinguished between the intermediate and complete uncertainty of the partially and fully occluded windows. These results are consistent with the existing literature showing that the ability to monitor and explicitly report uncertainty emerges and develops in the preschool years (e.g., Lyons \& Ghetti, 2013; Coughlin et al., 2015; Hembacher \& Ghetti, 2014) and extends this work to examine spontaneous judgments in the absence of training and feedback on how to use the confidence scale.

We predicted that observing evidence that disconfirms a current hypothesis would facilitate children's explicit recognition of uncertainty. Indeed, comparison of the first to last trials shows a marked improvement, but only for the younger children. On trial 1, 4-year-olds indicated that they were equally confident about all three windows. Following the experience of disconfirming evidence, their pattern of confidence ratings was indistinguishable from baseline performance of older children. Given that 5-year-olds did not similarly improve, this change is unlikely to be due to practice using the scale. Instead, these findings suggest that the experience of surprising or violating outcomes supports awareness of uncertainty when it is used to highlight the existence of incomplete knowledge.

\section{Experiment 2a}

In order to examine awareness of uncertainty in children's information search in a way that is appropriately matched to the procedure from Experiment 1, two additional experiments were needed: Experiment $2 \mathrm{a}$ examines baseline performance on a forced-choice exploration measure and Experiment $2 b$ examines the effect of disconfirming evidence on subsequent exploration behavior.

\section{Methods}

Participants Forty-five children, including 24 4-year-olds, ( $M=54.76$ months, $S D=3.64$, range: $48-58$ months) and 21 5-year-olds $(M=65.79$ months, $S D=3.68$, range: $60-72$ months) participated in Experiment 2a. An additional three children were excluded, due to experimenter error (2) or failure to complete the entire task (1). Children were recruited from the same locations with the same demographics as Experiment 1.

Materials A total of six paper windows (two Full, two Partial, two Clear) from Experiment 1 were used. The shapes (circle, heart, square, and rectangle), and their accompanying reference cards were also used.

Procedure The introduction and training trial were identical to Experiment 1, except that the confidence scale was not included.

As in Experiment 1, the experimenter began by laying out three windows (one of each level of occlusion), with the shapes already in place inside each. The target shape and window order were counterbalanced across participants. Again, the experimenter explained that some windows contained the shape displayed on the reference card, and some contained different shapes. However, instead of asking children to rate their confidence that each window contained the target shape, the experimenter offered children a choice to explore just one of the windows, saying: "Let's try to find out more about the shapes. We can look at what's behind just one of these windows. Which window should we look behind to find out more about the shapes?" If children did not spontaneously select a window to explore, they were prompted to point to the window of their choice. Once children made their choice, the experimenter revealed the shape and ended the session.

\section{Results}

The vast majority of children (88.98\%) spontaneously chose to explore one of the two uncertain windows at baseline. Indeed, children showed a significant preference to explore the window associated with the greatest amount of uncertainty, with $64.44 \%$ of children choosing the Full window, $X^{2}(2, N=45)=20.8, p<0.001$. Of the remaining children, $24 \%$ chose to explore the Partial window, and only $11.1 \%$ chose to explore the Clear window.

This pattern of behavior was also observed within each age group (see Figure 3). Chi-square tests revealed a significant preference to explore the full window in both 4year-olds $(62.5 \%), X^{2}(2, N=24)=9.75, p=0.007$, and 5year-olds $(66.7 \%), X^{2}(2, N=21)=11.14, p=0.003$, with no difference in distribution of exploration choices between age groups, $X^{2}(2, N=45)=0.219, p=0.896$.

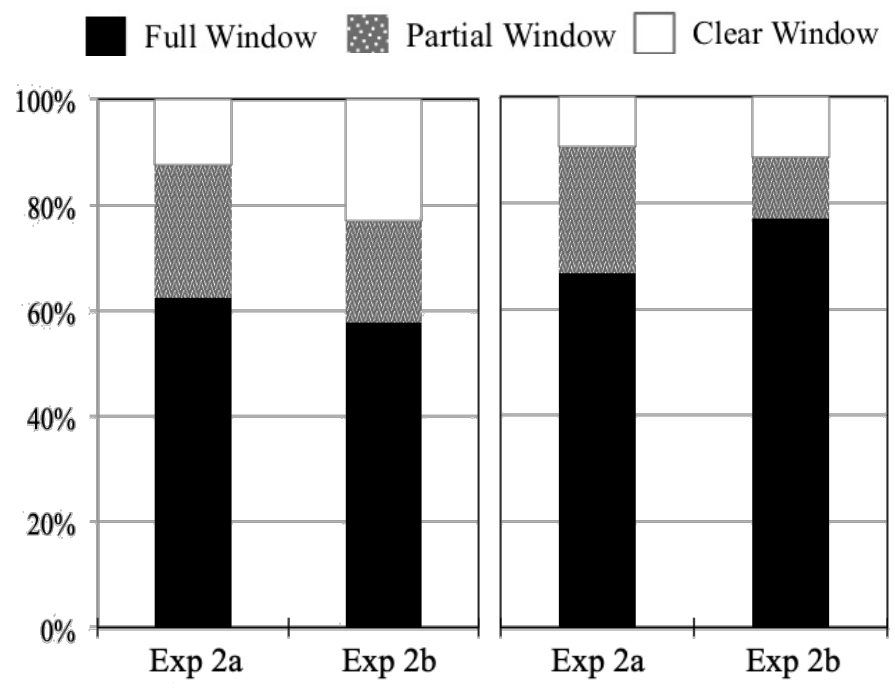

Figure 3. Exploration choices of four-year-olds (left) and five-year-olds (right) in Experiments $2 \mathrm{a}$ and $2 \mathrm{~b}$.

\section{Experiment 2b}

Due to the forced-choice format of Experiment 2a, children did not have the opportunity to observe the contents of all 
three windows. Experiment $2 b$ therefore recruited an additional sample of children to examine the effect of disconfirming evidence on exploration behavior. To do this, we largely replicated the procedure used in Experiment 1, except that we replaced the final confidence judgment (in Trial 3) with the exploration measure used in Trial 1 of Experiment 2a.

\section{Methods}

Participants Fifty-two children, including 26 4-year-olds, ( $M=54.86$ months, $S D=3.02$, range: $48-59$ months) and 26 5-year-olds $(M=64.97$ months, $S D=3.9$, range: $60-71$ months) participated in Experiment 2b. An additional 29 children were excluded, due to failure to pass the comprehension check (11), experimenter error (6), caregiver interference (9), or incomplete session (3). Children were recruited from the same locations with the same demographics as the previous experiments.

\section{Materials Materials were identical to Experiment 1.}

Procedure The training and first two test trials of Experiment 2b were identical to Experiment 1. On Trial 3, however, the final confidence judgment was replaced with a forced-choice exploration measure. As in Experiment 2a, the experimenter explained that, this time, children would only have the opportunity to reveal one shape, and offered a forced-choice among the three windows.

\section{Results}

There was no difference in the average age of children included in Experiments $2 \mathrm{a}$ and $2 \mathrm{~b}, t(90)=-0.003, p=$ $0.997(n s)$.

The pattern of children's exploration behavior following disconfirming evidence was similar to their baseline performance in Experiment 2a. Again, the majority of children $(82.69 \%)$ chose to explore one of the two uncertain windows, with $67.31 \%$ choosing the Full window, $X^{2}(2, N$ $=52)=27.04, p<0.001$. Of the remaining children, $15.4 \%$ chose to explore the Partial window and $17.3 \%$ chose to explore the Clear window.

This pattern of behavior was found in both 4-year-olds $(57.7 \%), X^{2}(2, N=26)=7, p=0.03$, and 5-year-olds $(76.9 \%), X^{2}(2, N=26)=22.23, p<0.001$, with no difference in the distribution of exploration choices between age groups, $X^{2}(2, N=52)=3.967, p=0.138(n s)$.

Comparison of the distribution of window choices between Experiments $2 \mathrm{a}$ and $2 \mathrm{~b}$ confirmed there was no difference in exploration behavior before and after observing disambiguating evidence, either overall $\left(X^{2}[2, N\right.$ $=97]=3.61, p=0.164)$ or within each age group (4-yearolds, $X^{2}[2, N=50]=2.77, p=0.25$, and 5-year-olds, $X^{2}[2$, $N=47]=2.16, p=0.339$ ).

\section{Discussion}

Experiments $2 \mathrm{a}$ and $2 \mathrm{~b}$ relied on a modified version of the procedure used in Experiment 1 to examine how 4-and- 5-year-olds would choose to explore the same ambiguous stimuli as an implicit measure of their sensitivity to uncertainty. The results of Experiment $2 \mathrm{a}$ are consistent with past work showing children's preference to explore ambiguous or surprising objects and events: The majority of younger and older children spontaneously chose to explore the window that would be most informative, and almost all children chose to explore where they had some amount of uncertainty. Critically, this contrasts with the baseline responses of younger children in Experiment 1, who did not make explicit confidence judgments that differentiated among the full, partial, and clear windows.

Furthermore, results of Experiment $2 \mathrm{~b}$ show no difference in exploration behavior following the same disconfirming evidence that served to improve 4-year-olds' explicit confidence judgments in Experiment 1. This suggests that, children are already intuitively aware of the presence of uncertainty in the context of exploration decisions.

\section{General Discussion}

This study examined both confidence scale judgments and exploration to compare explicit and implicit measures of children's sensitivity to uncertainty. We also examined the use of disconfirming evidence to highlight the presence of incomplete knowledge.

In Experiment 1, we looked at children's explicit confidence judgments in the absence of task-specific training or feedback, and asked whether they would improve following surprising events. In line with prior work, children's explicit ratings generally tended towards overconfidence. However, by age 5, children accurately report their awareness of relative differences in uncertainty. Even at baseline, 5-year-olds judgments reflected their recognition of the difference, not only between complete information and complete ambiguity, but also between complete and incomplete evidence. Four-year-olds did not make these distinctions spontaneously. However, our results indicate that the presentation of disconfirming evidence can facilitate younger children's recognition of uncertainty.

The effect of this modest intervention on younger children's performance suggests that implicit sensitivity to surprising events may extend to impact the development of uncertainty monitoring. Specifically, when the experimenter revealed an irregular shape from behind the partially occluded window, this evidence violated children's existing belief that the window contained the target-shape. This experience likely highlighted the uncertainty associated with the Partial window, impacting future trials. This novel paradigm may therefore provide an effective training tool to facilitate children's ability to accurately report their own confidence in both research and applied settings.

We also investigated children's implicit sensitivity to uncertainty, by examining their exploration decisions using the same stimuli in Experiments $2 \mathrm{a}$ and $2 \mathrm{~b}$. Past work has suggested that decision-making may demonstrate children's implicit awareness of their own uncertainty in some domains (e.g., Ghetti et al., 2013; Hembacher \& Ghetti, 
2014). However, to our knowledge, this is the first study to compare children's information-seeking decisions as a measure of uncertainty control in direct comparison to confidence scale use on the same task. We found that both 4- and 5-year-olds showed sensitivity to their own uncertainty, by preferentially choosing to explore where they had incomplete information, even before the presentation of disconfirming evidence. This early implicit sensitivity to uncertainty is consistent with the prior work on self-directed learning reviewed above (e.g., Legare, 2012; Schulz \& Bonawitz, 2007; Bonawitz, et al., 2012).

Critically, these findings provide evidence that children implicitly recognize what is most likely to be informative well before they are able to explicitly articulate that understanding (e.g., Cook, et al., 2011; Lapidow \& Walker, 2019). This suggests that children's decision-making during information search may be an early developing form of implicit uncertainty control and provide a robust measure of children's recognition of what they don't know.

\section{Acknowledgments}

This work was supported by the Hellman Fellowship to Caren Walker, and the NDSEG Fellowship to Elizabeth Lapidow. Special thanks to Cesia Rojas, Paul Simental, Sally Tang, and Nicky Sullivan for their efforts towards data collection.

\section{References}

Beck, S. R., McColgan, K. L. T., Robinson, E. J., \& Rowley, M. G. (2011). Imagining what might be: Why children underestimate uncertainty. Journal of Experimental Child Psychology, 110(4), 603-610.

Beran, M. J., Smith, J. D., \& Perdue, B. M. (2013). Language-Trained Chimpanzees (Pan troglodytes) Name What They Have Seen but Look First at What They Have Not Seen. Psychological Science 24(5), 660-666.

Bonawitz, E. B., van Schijndel, T. J. P., Friel, D., \& Schulz, L. (2012). Children balance theories and evidence in exploration, explanation, and learning. Cognitive Psychology, 64(4), 215-234.

Call, J., \& Carpenter, M. (2001). Do apes and children know what they have seen? Animal Cognition, 3(4), 207-220.

Cook, C., Goodman, N. D., \& Schulz, L. E. (2011). Where science starts: spontaneous experiments in preschoolers' exploratory play. Cognition, 120(3), 341-349.

Coughlin, C., Hembacher, E., Lyons, K. E., \& Ghetti, S. (2015). Introspection on uncertainty and judicious helpseeking during the preschool years. Developmental Science, 18(6), 957-971.

Destan, N., Hembacher, E., Ghetti, S., \& Roebers, C. M. (2014). Early metacognitive abilities: The interplay of monitoring and control processes in 5- to 7-year-old children. Journal of Experimental Child Psychology 126, 213-228.
Ghetti, S., H embacher, E., \& Coughlin, C. A. (2013). Feeling uncertain and acting on it during the preschool years: A metacognitive approach. Child Development Perspectives, 7(3), 160-165.

Hembacher, E., \& Ghetti, S. (2014). Don't Look at My Answer: Subjective Uncertainty Underlies Preschoolers' Exclusion of Their Least Accurate Memories. Psychological Science 25(9), 1768-1776.

Kidd, C., Piantadosi, S. T., \& Aslin, R. N. (2014). The goldilocks effect in infant auditory attention. Child Development, 85(5), 1795-1804.

Koriat, A., \& Goldsmith, M. (1996). Monitoring and Control Processes in the Strategic Regulation of Memory Accuracy. Psychological Review 103(3), 490.

Lapidow, E., \& Walker, C. M. (2020). Informative experimentation in intuitive science: Children select and learn from their own causal interventions. Cognition, 201, 104315.

Legare, C. H. (2012). Exploring explanation: Explaining inconsistent evidence informs exploratory, hypothesistesting behavior in young children. Child Development 83(1), 173-185.

Lyons, K. E., \& Ghetti, S. (2011). The Development of Uncertainty Monitoring in Early Childhood. Child Development 82(6), 1778-1787.

Lyons, K. E., \& Ghetti, S. (2013). I Don't Want to Pick! Introspection on Uncertainty Supports Early Strategic Behavior. Child Development 84(2), 726-736.

Marsh, H. L., \& Macdonald, S. E. (2012). Orangutans (Pongo abelii) "play the odds": Information-seeking strategies in relation to cost, risk, and benefit. Journal of Comparative Psychology, 126(3), 263.

Newman, R. S., \& Wick, P. L. (1987). Effect of Age, Skill, and Performance Feedback on Children's Judgments of Confidence. Journal of Educational Psychology, 79(2), 115.

Paukner, A., Anderson, J. R., \& Fujita, K. (2006). Redundant food searches by capuchin monkeys (Cebus apella): A failure of metacognition? Animal Cognition, 9(2), 110-117.

Roebers, C. M. (2002). Confidence judgments in children's and adults' event recall and suggestibility. Developmental Psychology, 85(4), 352-371.

Rohwer, M., Kloo, D., \& Perner, J. (2012). Escape From Metaignorance: How Children Develop an Understanding of Their Own Lack of Knowledge. Child Development, 83(6), 1869-1883.

Schulz, L. E., \& Bonawitz, E. B. (2007). Serious Fun: Preschoolers Engage in More Exploratory Play When Evidence Is Confounded. Developmental Psychology, 43(4), 1045.

Stahl, A. E., \& Feigenson, L. (2015). Observing the unexpected enhances infants' learning and exploration. Science, 348(6230), 91-94. 